\title{
PARTITIONS WITH EQUAL PRODUCTS (II)
}

\author{
JOHN B. KELLY
}

(Communicated by William Adams)

\begin{abstract}
The following theorem is proved: Let $k \geq 3$ and $r$ be positive integers. There exist infinitely many integers having $r$ partitions into $k$ parts such that the products of the integers in each partition are equal. Moreover, these partitions are mutually disjoint, i.e., no integer occurs in more than one of them.

Of some additional interest is a lemma stating that a certain class of elliptic curves has positive rank over $\mathbf{Q}$.
\end{abstract}

\section{INTRODUCTION}

In [1] we proved the following theorem:

Theorem 1. Let $k$ be a positive integer greater than or equal to 3 . There exists an integer $N(k)$ such that every integer greater than $N(k)$ has $(k-1)$ partitions into $k$ parts such that the products of the integers in each partition are equal. Moreover, these partitions are mutually disjoint, i.e., no integer occurs in more than one of them.

Examples such as

$$
\begin{gathered}
76+28+27=72+38+21=57+56+18=131 \\
76 \cdot 28 \cdot 27=72 \cdot 38 \cdot 21=57 \cdot 56 \cdot 18=57456
\end{gathered}
$$

show that in some cases more than $k-1$ partitions into $k$ parts with equal products are possible. Here we prove a theorem which is stronger than Theorem 1 in the sense that $k-1$ is replaced by an arbitrary integer but weaker in the sense that it does not hold for all sufficiently large integers but only for infinitely many.

Theorem 2. Let $k \geq 3$ and $r$ be positive integers. There exist infinitely many integers having $r$ mutually disjoint partitions into $k$ parts with equal products.

Note that the general case of Theorem 2 would be a trivial consequence of the case $k=3$ if we did not impose the requirement of mutual disjointness. For

Received by the editors October 24, 1988 and, in revised form, January 22, 1989.

1980 Mathematics Subject Classification (1985 Revision). Primary 11P80; Secondary 11G05.

Key words and phrases. Partition, elliptic curve, torsion group. 
suppose that the integer $N$ had $r$ partitions into 3 parts with equal products. By adjoining a set of $(k-3)$ 1's to each of these $r$ partitions we would obtain $r$ partitions of $N+k-3$ into $k$ parts with equal products.

Our proof of Theorem 2 is elementary except for the use of Mazur's theorem, [2], [3], on the torsion group of an elliptic curve in establishing Lemma 1. This lemma, as well as Theorem 3 , concerning partitions into rational parts with equal products, may be of independent interest.

\section{Proof of Theorem 2}

Lemma 1. Let $a_{1}, a_{2}$ and $a_{3}$ be positive rational numbers with $a_{1}>a_{2}>a_{3}$ and

$$
\begin{gathered}
a_{1}+a_{2}+a_{3}=A \\
a_{1} a_{2} a_{3}=B .
\end{gathered}
$$

Suppose further that

$$
a_{1}\left(a_{2}-a_{3}\right)^{3} \neq a_{3}\left(a_{1}-a_{2}\right)^{3} .
$$

Then the diophantine system

$$
\begin{gathered}
x_{1}+x_{2}+x_{3}=A, \\
x_{1} x_{2} x_{3}=B .
\end{gathered}
$$

has infinitely many positive rational solutions.

Proof. If we set $x_{1}=x, x_{2}=y$, we see that (3) is equivalent to the equation

$$
x y(A-x-y)=B
$$

which determines a cubic curve $\Gamma$. The critical points of $x y(A-x-y)$ are $(A / 3, A / 3),(0,0),(A, 0)$ and $(0, A)$. The last three are not on $\Gamma$ since $B>0$. If $(A / 3, A / 3)$ satisfied (4), then we should have $B=A^{3} / 27$ which implies, from (1), that

$$
\frac{a_{1}+a_{2}+a_{3}}{3}=\sqrt[3]{a_{1} a_{2} a_{3}} .
$$

Equality holds in the arithmetic-geometric mean inequality only if $a_{1}=a_{2}=$ $a_{3}$, contrary to our hypothesis. Thus $\Gamma$ is a cubic curve without a singular point, that is, a curve of genus 1 . Since $\Gamma$ has a rational point $\left(a_{1}, a_{2}\right)$, it is elliptic over the rational field $\mathbf{Q}$.

The real affine curve with equation (4) is displayed in Figure 1. Its three unbounded components $\Gamma_{1}, \Gamma_{2}$ and $\Gamma_{3}$ are convex with the coordinate axes and the line $x+y=A$ as asymptotes; the bounded component $\Gamma_{0}$ is a closed convex curve. There are six rational points on $\Gamma_{0}$, namely $P_{1}\left(a_{1}, a_{2}\right)$, $P_{2}\left(a_{1}, a_{3}\right), P_{3}\left(a_{2}, a_{3}\right), P_{4}\left(a_{3}, a_{2}\right), P_{5}\left(a_{3}, a_{1}\right)$ and $P_{6}\left(a_{2}, a_{1}\right)$ taken in clockwise order around $\Gamma_{0}$. If $\Gamma$ is regarded as a curve in the projective plane with homogeneous equation

$$
x^{2} y+y^{2} x-A x y z+B z^{3}=0
$$




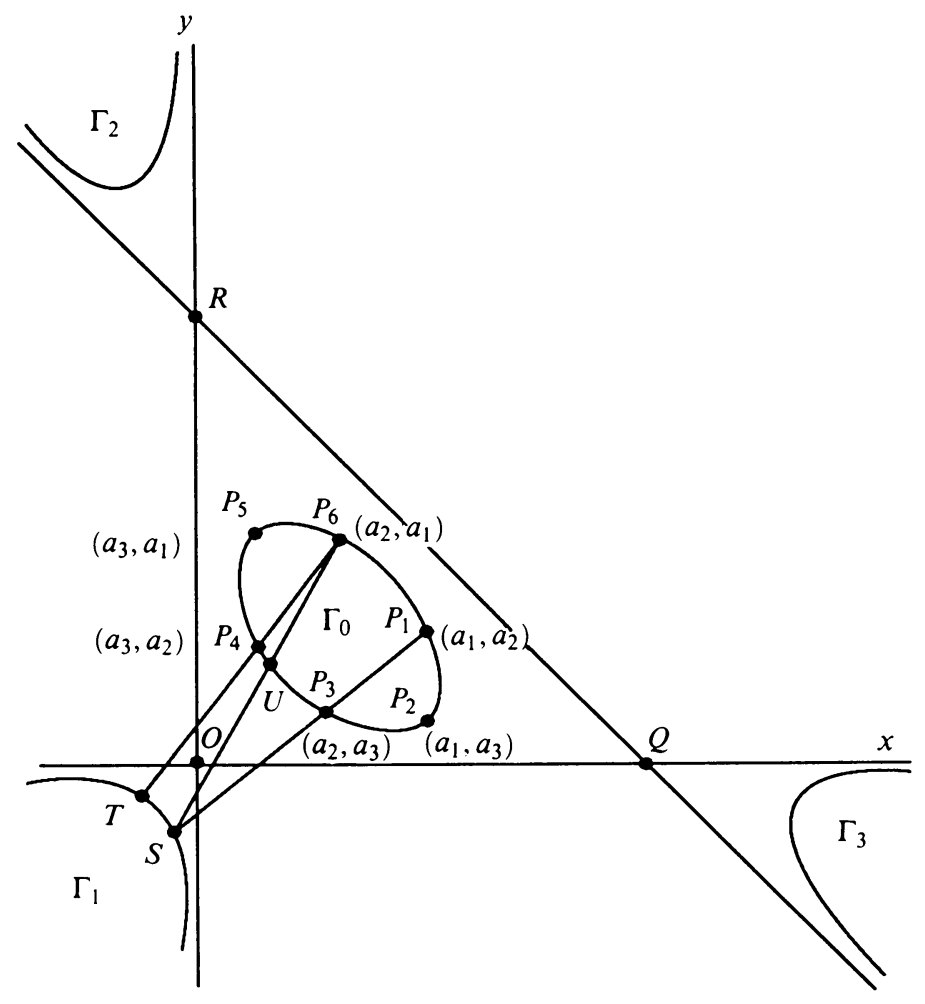

FIGURE 1.

it contains, in addition, three rational points "at infinity," $(1,0,0),(0,1,0)$ and $(1,-1,0)$, corresponding to its asymptotes.

Lines joining certain pairs of the six rational points on $\Gamma_{0}$ will intersect $\Gamma_{1}$, $\Gamma_{2}$, and $\Gamma_{3}$ in additional rational points. It is routine to calculate that $P_{1} P_{3}$ meets $\Gamma_{1}$ at point $S$ with coordinates

$$
\left(\frac{-a_{3}\left(a_{1}-a_{2}\right)^{2}}{\left(a_{2}-a_{3}\right)\left(a_{1}-a_{3}\right)}, \frac{-a_{1}\left(a_{2}-a_{3}\right)^{2}}{\left(a_{1}-a_{2}\right)\left(a_{1}-a_{3}\right)}\right) \text {. }
$$

Similarly $P_{4} P_{6}$ meets $\Gamma_{1}$ at point $T$ with coordinates

$$
\left(\frac{-a_{1}\left(a_{2}-a_{3}\right)^{2}}{\left(a_{1}-a_{2}\right)\left(a_{1}-a_{3}\right)}, \frac{-a_{3}\left(a_{1}-a_{2}\right)^{2}}{\left(a_{2}-a_{3}\right)\left(a_{1}-a_{3}\right)}\right) \text {. }
$$

These two points coincide if and only if

$$
a_{3}\left(a_{1}-a_{2}\right)^{3}=a_{1}\left(a_{2}-a_{3}\right)^{3}
$$

which is ruled out by (2). In the same manner we can find two more rational points on each of $\Gamma_{2}$ and $\Gamma_{3}$. From Figure 1 it is clear, for example, that, since $P_{1} P_{6}$ and $P_{3} P_{4}$ are parallel to $R Q, P_{3} P_{5}$ and $P_{2} P_{6}$ must intersect $\Gamma_{2}$. The condition that the two points of intersection be distinct turns out again to be (2). Likewise, $P_{2} P_{4}$ and $P_{1} P_{5}$ intersect $\Gamma_{3}$ in distinct points. 
$S P_{6}$ meets $\Gamma_{0}$ at the rational point $U$ on the shorter arc of $\Gamma_{0}$ strictly between $P_{3}$ and $P_{5}$ because $P_{3} P_{6}$ and $P_{5} P_{6}$ are parallel to the asymptotes of $\Gamma_{1}$. (This reasoning is valid regardless of the position of the intersection of $P_{1} S$ and $P_{6} T$ relative to $\Gamma_{1}$.) $U$ and $P_{4}$ cannot coincide since $U$ is on $P_{6} S, P_{4}$ is on $P_{6} T$ and $S \neq T$. Similarly there are additional rational points on the shorter arcs of $\Gamma_{0}$ joining $P_{5}$ and $P_{1}$, and $P_{1}$ and $P_{3}$. Thus we have accumulated, including the three points at infinity, a total of eighteen rational points on $\Gamma$.

Since $\Gamma$ is an elliptic curve, it may be given the structure of an algebraic variety. Mordell's theorem states that the group of rational points on $\Gamma$ is a finitely generated abelian group. Mazur's theorem [2], [3] states that the torsion subgroup of this group is isomorphic to one of $\mathbf{Z}_{i}, 1 \leq i \leq 10, \mathbf{Z}_{12}, \mathbf{Z}_{2} \times \mathbf{Z}_{2}$, $\mathbf{Z}_{2} \times \mathbf{Z}_{4}, \mathbf{Z}_{2} \times \mathbf{Z}_{6}$ and $\mathbf{Z}_{2} \times \mathbf{Z}_{8}$. The largest of these groups, $\mathbf{Z}_{2} \times \mathbf{Z}_{8}$ has sixteen points. Since $\Gamma$ has more than sixteen rational points, not all of them can be of finite order and there must be infinitely many of them.

If there were only finitely many rational points on $\Gamma_{0}$, there would have to be infinitely many on at least one of the unbounded components. The lines joining each of these points with $P_{1}$ would intersect $\Gamma_{0}$ in infinitely many distinct rational points, a contradiction. Thus (3) has infinitely many positive rational solutions.

Corollary 1. Under the hypotheses of Lemma 1, there exist infinitely many positive rational solutions of (3) with denominator exceeding any pre-assigned positive number, $M$.

Proof. For any positive rational solution of $x_{1}+x_{2}+x_{3}=A$ we have $0<x_{i}<$ $A, 1 \leq i \leq 3$. It follows that the number of possible values of the $x_{i}$ with denominator $\leq M$ is finite. There is essentially, i.e., apart from permutations, one solution of (3) in which a given number, say $a$, occurs as the value of one of the unknowns, say $x_{1}$. For the system

$$
x_{2} x_{3}=B / a, \quad x_{2}+x_{3}=A-a
$$

has only one solution, apart from permutations. Hence there are only finitely many solutions of (3) in positive rationals in which one or more of the unknowns have denominator $\leq M$. Therefore, by Lemma 1 , there are infinitely many solutions in which all of the unknowns have denominators in excess of $M$.

The system

$$
\begin{gathered}
x+y+z=37, \\
x y z=720
\end{gathered}
$$

has the solution $(24,10,3)$ which violates (2). Professor Andrew Bremner has shown that this system has only finitely many rational solutions, viz., 6 permutations of $(24,10,3)$, three permutations of $(45,-4,-4)$ and three points at infinitely (arising when $z$ is eliminated). So Lemma 1 would be invalid if condition (2) were removed and not replaced by something else. 
Next, we examine the consequences of (2) more closely. Let $\sigma=$ $\left\{s_{1}, s_{2}, s_{3}\right\}$ be a triple of real numbers with $s_{1} \geq s_{2} \geq s_{3}$. We define

$$
F(\sigma)=s_{1}\left(s_{2}-s_{3}\right)^{3}-s_{3}\left(s_{1}-s_{2}\right)^{3} \text {. }
$$

Lemma 2. Let $\tau=\left\{t_{1}, t_{2}, t_{3}, t_{4}\right\}$ be a set of four distinct positive numbers. Define $\tau_{i}=\tau-\left\{t_{i}\right\}, 1 \leq i \leq 4$. At most two of the four quantities $F\left(\tau_{i}\right)$, $1 \leq i \leq 4$, can vanish.

Proof. We may assume that $t_{1}>t_{2}>t_{3}>t_{4}>0$. Clearly $F\left(\tau_{3}\right)>F\left(\tau_{4}\right)$ and $F\left(\tau_{3}\right)>F\left(\tau_{2}\right)$ so if $F\left(\tau_{3}\right) \leq 0$, the lemma is evident. If $F\left(\tau_{3}\right)>0$ and $F\left(\tau_{1}\right)=F\left(\tau_{2}\right)=0$ we have

$$
\frac{\left(t_{3}-t_{4}\right)^{3}}{t_{4}}=\frac{\left(t_{1}-t_{3}\right)^{3}}{t_{1}}=\frac{\left(t_{2}-t_{3}\right)^{3}}{t_{2}},
$$

whence

$$
t_{1}\left(t_{2}-t_{3}\right)^{3}=t_{2}\left(t_{1}-t_{3}\right)^{3}
$$

so that

$$
F\left(\tau_{4}\right)=t_{2}\left(t_{1}-t_{3}\right)^{3}-t_{3}\left(t_{1}-t_{2}\right)^{3}>0 \text {. }
$$

Lemma 3. Let $a_{1}, a_{2}, a_{3}, a_{4}$ be distinct positive rational numbers such that

$$
\begin{gathered}
a_{1}+a_{2}+a_{3}+a_{4}=A, \\
a_{1} a_{2} a_{3} a_{4}=B .
\end{gathered}
$$

Let $M$ be a positive number. There exist distinct positive rational numbers $b_{1}$, $b_{2}, b_{3}, b_{4}$, all with denominator greater than $M$, such that

$$
\begin{gathered}
b_{1}+b_{2}+b_{3}+b_{4}=A, \\
b_{1} b_{2} b_{3} b_{4}=B .
\end{gathered}
$$

Proof. By Lemma 2 we may assume that $a_{1}>a_{2}>a_{3}>0$ and

$$
a_{1}\left(a_{2}-a_{3}\right)^{3} \neq a_{3}\left(a_{1}-a_{2}\right)^{3} \text {. }
$$

Corollary 1 tells us that the system

$$
\begin{gathered}
x_{1}+x_{2}+x_{3}=A-a_{4}, \\
x_{1} x_{2} x_{3}=B / a_{4}
\end{gathered}
$$

has infinitely many positive rational solutions with denominator greater than $M$. The number of solutions with two of the unknowns equal is finite as is the number with one of them equal to $a_{4}$. Hence we can find a solution $\left(d_{1}, d_{2}\right.$, $d_{3}$ ) with the $d_{i}$ positive rationals distinct from each other and from $a_{4}$ and having denominators greater than $M$. Applying Lemma 2, we conclude that $F$ must fail to vanish on some 3 -subset of $\left\{d_{1}, d_{2}, d_{3}, a_{4}\right\}$ containing $a_{4}$. Assume then, that $F\left(\left\{d_{1}, d_{2}, a_{4}\right\}\right) \neq 0$. Note that the system

$$
\begin{gathered}
x_{1}+x_{2}+x_{3}=A-d_{3}, \\
x_{1} x_{2} x_{3}=B / d_{3}
\end{gathered}
$$


has solution $\left(d_{1}, d_{2}, a_{4}\right)$. Reasoning as before from Corollary 1 , we see that the system (6) has a solution $\left(e_{1}, e_{2}, e_{3}\right)$ in positive rationals which are distinct from each other and from $d_{3}$ and which have denominators greater than $M$. Setting $b_{1}=e_{1}, b_{2}=e_{2}, b_{3}=e_{3}, b_{4}=d_{3}$ completes the proof of Lemma 3.

Lemma 4. Let $k \geq 4$. Let $a_{1}, \ldots, a_{k}$ be distinct positive rationals such that

$$
a_{1}+\cdots+a_{k}=A, \quad a_{1} \cdots a_{k}=B .
$$

Let $M$ be a positive number. There exist distinct positive rationals $b_{1}, \cdots, b_{k}$ with denominators greater than $M$ such that

$$
b_{1}+\cdots+b_{k}=A, \quad b_{1} \cdots b_{k}=B .
$$

Proof. We proceed by induction on $k$. The case $k=4$ is the previous lemma. Assume the lemma for $k=n$ and let $a_{1}, a_{2}, \ldots, a_{n+1}$ be $n+1$ distinct positive rationals such that

$$
a_{1}+\cdots+a_{n+1}=A, \quad a_{1} \cdots a_{n+1}=B .
$$

Let $M^{\prime}$ be the denominator of $a_{n+1}$ and let $M^{*}=\max \left(M, M^{\prime}\right)$. By the inductive hypothesis there exist distinct positive rationals $d_{1}, \ldots, d_{n}$ with denominator $>M^{*}$ such that

$$
d_{1}+\cdots+d_{n}=A-a_{n+1}, \quad d_{1} \cdots d_{n}=B / a_{n+1} .
$$

These rationals are also distinct from $a_{n+1}$, having a higher denominator. Note that

$$
d_{1}+\cdots+d_{n}+a_{n+1}=A, \quad d_{1} d_{2} \cdots d_{n} a_{n+1}=B .
$$

Now consider the system

$$
\begin{gathered}
x_{1}+\cdots+x_{n+1}=A-d_{1}, \\
x_{2} \cdots x_{n+1}=B / d_{1} .
\end{gathered}
$$

(7) has the solution $x_{i}=d_{i}, 2 \leq i \leq n, x_{n+1}=a_{n+1}$ in distinct positive rationals. Let $M^{\prime \prime}$ be the denominator of $d_{1}$. Applying the inductive hypothesis again, we see that (7) has a solution $x_{i}=b_{i}, 2 \leq i \leq n+1$ in distinct positive rationals with denominator $>M^{\prime \prime}>M$. These rationals are distinct from $d_{1}$, having a higher denominator. Setting $b_{1}=d_{1}$ establishes the case $k=n+1$ and completes the proof of the lemma.

Theorem 3. Let $k \geq 3$. Let $a_{1}, a_{2}, \ldots, a_{k}$ be distinct positive rationals such that

$$
a_{1}+a_{2}+\cdots+a_{k}=A, \quad a_{1} a_{2} \cdots a_{k}=B .
$$

If $k=3$, assume in addition, that

$$
F\left(\left\{a_{1}, a_{2}, a_{3}\right\}\right) \neq 0
$$

then the system

$$
x_{1}+x_{2}+\cdots+x_{k}=A, \quad x_{1} x_{2} \cdots x_{k}=B
$$


has an infinite family of solutions in distinct positive rationals such that no rational number occurs in more than one solution of the family.

Proof. Repeatedly apply Corollary 1 if $k=3$ and Lemma 4 if $k \geq 4$. At the $n$th step let $M=M_{n}=$ maximum denominator of all previously obtained solutions. In the case $k=3$ we also use the fact that the system

$$
x_{1}+x_{2}+x_{3}=A, \quad x_{1} x_{2} x_{3}=B
$$

has only finitely many solutions in which the unknowns are not distinct.

Lemma 5. Let $k$ and $\ell$ be positive integers with $k \geq 3$. There exists an integer $N(k, \ell)$ having $\ell$ mutually disjoint partitions into $k$ parts, all with the same product.

Proof. Let $A$ and $B$ be two rationals to which Theorem 3 applies. Find $\ell$ positive rational solutions of the system

$$
\begin{gathered}
x_{1}+\cdots+x_{k}=A, \\
x_{1} \cdots x_{k}=B
\end{gathered}
$$

so that no rational number occurs in more than one of them. Let $D$ be the least common multiple of all the denominators in all these solutions. Let $N(k, \ell)=$ $A D$. The system

$$
\begin{gathered}
y_{1}+\cdots+y_{k}=A D, \\
y_{1} \cdots y_{k}=B D^{k}
\end{gathered}
$$

has $\ell$ mutually disjoint integral solutions obtained by putting $y_{i}=D x_{i}$, $1 \leq i \leq k$ in each of the $\ell$ solutions of (8).

Proof of Theorem 2. The set

$$
N_{k, r}=\{N(k, \ell): \ell \geq r\}
$$

contains infinitely many integers since any particular integer has only a finite number of partitions with $k$ parts. Any integer in $N_{k, r}$ satisfies the conclusion of Theorem 2.

\section{REFERENCES}

1. J. B. Kelly, Partitions with equal products, Proc. Amer. Math. Soc. 15 (1964), 987-990.

2. B. Mazur, Modular curves and the Eisenstein ideal, Publ. Math. I.H.E.S. 47 (1977), 33-186.

3. Rational isogenies of prime degree, Inv. Math 44 (1978), 129-162. 